\title{
Lupus erythematosus with leflunomide: induction or reactivation?
}

\author{
D Gensburger, M Kawashima, H Marotte, J Kanitakis, P Miossec
}

Ann Rheum Dis 2005;64:153-155. doi: 10.1136/ard.2003.019323

Background: Leflunomide is a new oral disease modifying antirheumatic drug with a good safety profile.

Case report: The first case of lupus erythematosus in a 58 year old white woman after administration of leflunomide for primary Siögren's syndrome is reported. The relationship between induced lupus and leflunomide was confirmed by the resolution of the skin rash when the drug was stopped and its recurrence when it was reintroduced following a dose-response effect.

Discussion: Peripheral blood cells from this patient, from 15 patients with rheumatoid arthritis, and from healthy controls were used in a bioassay, which suggested that leflunomide affected the Th1/Th2 balance. Such a side effect might be related, in part, to the anti-tumour necrosis factor $\alpha$ activity of leflunomide.

L eflunomide is now approved for the treatment of rheumatoid arthritis (RA) and has a good safety profile. ${ }^{12}$ Common adverse events reported during drug trials included gastrointestinal symptoms, reversible alopecia, transient alterations in liver function tests, and hypertension. ${ }^{3}$ Unusual side effects have been recently reported with its increased clinical use, such as vasculitis, ${ }^{4}$ skin ulceration, ${ }^{5}$ and peripheral neuropathy. ${ }^{6}$ We present here the first case, to our knowledge, of lupus erythematosus after leflunomide administration, showing not only a temporal relationship but also a positive dose-response effect. A reintroduction further demonstrated the involvement of the drug, allowing a study of its mode of action.

\section{CASE REPORT}

This 58 year old white woman had an 11 year history of inflammatory disease. A diagnosis of primary Sjögren's syndrome had been made in 1992 on inflammatory nonerosive arthritis and major salivary gland hypertrophy leading to bilateral parotidectomy. A pathological study concluded that it was myoepithelial sialadenitis without features of lymphoma. Other laboratory findings at the onset of disease included positive rheumatoid factor, low titre (1/256) antinuclear antibodies, negative anti-DNA antibodies, and positive anti-extractable nuclear antigen (ENA; SSA and SSB) antibodies. Levels of C3 and C4 were normal. HLA typing was Al/A32; B8/B63; DR3/DR13/DR52; DQ6/DQ7.

Because of active arthritis, the patient was treated with methotrexate for 8 years with increasing doses up to $15 \mathrm{mg} /$ week. Owing to poor efficacy, methotrexate was stopped and leflunomide was started in September 2000 at a dose of $100 \mathrm{mg}$ for 3 days, followed by $20 \mathrm{mg}$ daily. One month later, despite a clear positive effect on joint manifestations, she developed a malar lupus skin reaction. Laboratory data showed an increased titre of homogeneous antinuclear antibodies (1/2560). Anti-DNA and antihistone antibodies were negative, C3 and C4 levels were normal, and anti-ENA antibodies (anti-SSA and anti-SSB) were positive. A skin biopsy showed features of discoid lupus erythematosus (fig lC). Leflunomide was discontinued, the skin rash completely resolved within 4 weeks and the antinuclear titre decreased to $1 / 640$. There were no other clinical or biological lupus manifestations-in particular, no nephropathy.

During the following 2 years, the patient was treated with azathioprine, cyclophosphamide, and infliximab combined with methotrexate, but these drugs had to be discontinued because of lack of efficacy or poor tolerance. After pressure from the patient who considered leflunomide to be the most active drug for her arthritis symptoms, this treatment was reintroduced in January 2003 at a low dose of $10 \mathrm{mg}$ daily.

Three months later, she was free of skin lesions but noted no improvement in her arthritis. With her consent, leflunomide was increased to $20 \mathrm{mg}$. When seen 3 months later in July 2003, she presented an acute lupus-like skin rash over the face, neck and arms (fig lA) that had appeared 8 days after the increase to $20 \mathrm{mg}$. Moreover, she now had leucopenia, increased titre of antinuclear antibodies and anti-SSA and anti-SSB antibodies, but antihistone antibodies were negative. A new skin biopsy showed, in addition to epidermal atrophy, a lymphocytic infiltration suggestive of subacute lupus (fig 1D). Immunofluorescence of that skin biopsy showed deposits of $\operatorname{IgM}$ and $\mathrm{C} 3$ at the dermoepidermic junction. Again, there were no other signs of lupus. Leflunomide was stopped and the skin rash resolved within four weeks.

One month later, although she was strongly advised not to take the drug, she took again $10 \mathrm{mg}$ a day and quickly developed a severe skin eruption, again typical of subacute lupus (fig 1B). These manifestations disappeared with oral steroids. At this stage, the patient reported that the skin manifestations were identical to those seen at the onset of disease. At that time, she was followed up at the department of dermatology with the diagnosis of cutaneous lupus. However, anti-DNA antibodies were negative and major salivary gland hypertrophy led to bilateral parotidectomy, suggesting that she had Sjögren's syndrome.

\section{MATERIALS AND METHODS}

To explore the systemic immune function, a bioassay based on the induction of interferon $\gamma$ (IFN $\gamma$ ) production by interleukin (IL)12 and/or IL18 was carried out with peripheral blood mononuclear cells (PBMC) from this patient, 15 patients with RA, and 14 healthy controls. ${ }^{7}$ PBMC from this patient were collected in July 2003, while the patient was having the second skin reaction. PBMC were isolated by Ficoll and stimulated or not by ILl2 ( $1 \mathrm{ng} / \mathrm{ml}$, R\&D systems, Abingdon, UK) and IL18 (5 ng/ml, MBL, Nagoya, Japan) for

Abbreviations: ELISA, enzyme linked immunosorbent assay; ENA, extractable nuclear antigen; IFN $\gamma$, interferon $\gamma$; IL, interleukin; PBMC, peripheral blood mononuclear cells; RA, rheumatoid arthritis; TNF $\alpha$, tumour necrosis factor $\alpha$ 

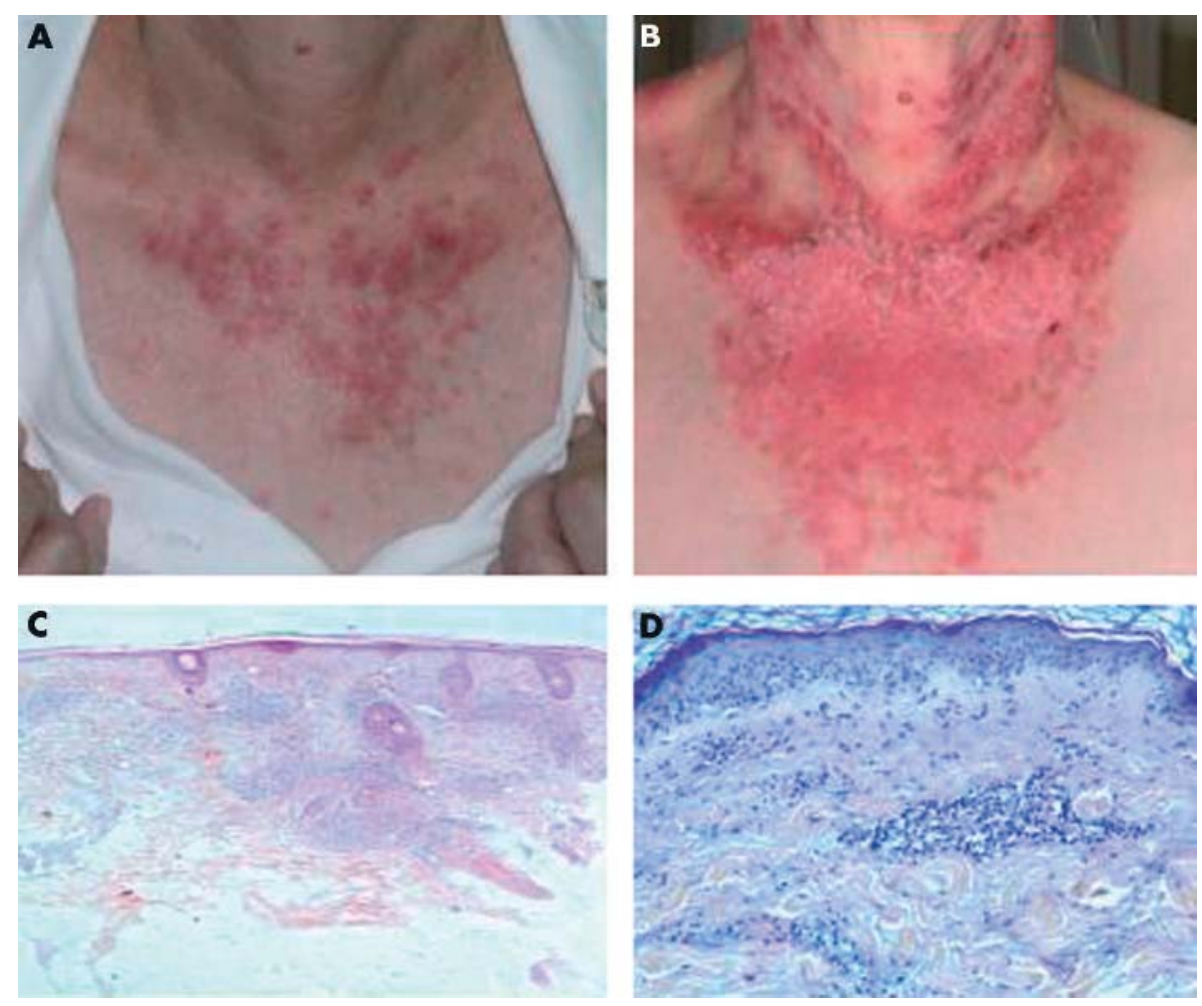

Figure 1 Skin clinical and pathology changes: a typical lupus skin rash seen in July 2003 (A) and in September 2003 (B). Skin biopsy in November 2000 (face) showing signs of discoid lupus (C) and in July 2003 (arm) showing signs of subacute lupus (D).

7 days. IFN $\gamma$ was measured by enzyme linked immunosorbent assay (ELISA; DuoSet ELISA Development System human IFN $\gamma$, R\&D Systems).

\section{RESULTS AND DISCUSSION}

This is, to our knowledge, the first case of lupus related to leflunomide. Other autoimmune disorders reported with leflunomide include a few cases of vasculitis ${ }^{5}$ and of peripheral neuropathy ${ }^{7}$ possibly related to vasculitis.

In this patient the role of leflunomide is based on the following criteria: a temporal relationship seen on two

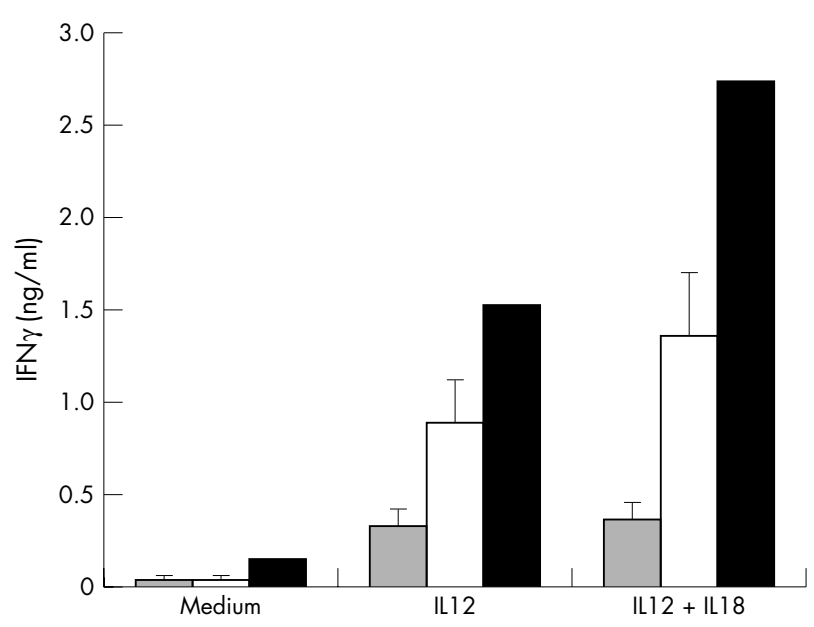

Figure 2 Th1 immune modulation in patients with RA, healthy controls, and our patient. PBMC from the patient (black bar), control patients with RA ( $n=15$, grey bar), and healthy controls ( $n=14$, white bar), were stimulated with or without $1 \mathrm{ng} / \mathrm{ml}$ IL12 or a combination of IL12 (1 ng/ $\mathrm{ml}$ ) and ILI $8(5 \mathrm{ng} / \mathrm{ml})$. IFN $\gamma$ concentrations in culture supernatants were measured by ELISA. occasions, resolution when the drug was stopped, reappearance of the rash with reintroduction of the drug following a dose-response relationship, and the absence of other candidate drugs. In this case, it should be indicated that the diagnosis was primary Sjögren's syndrome rather than RA, for which the drug has been registered. Because the patient later described the same rash as at the beginning of her disease, this case is rather a reactivation than an induction of lupus.

The mode of action of leflunomide is not completely known. ${ }^{8}$ Its active metabolite A771 726 inhibits the mitochondrial enzyme dihydro-orotate dehydrogenase and therefore inhibits the de novo synthesis of pyrimidine in immune cells, particularly in activated T cells. Furthermore, leflunomide inhibits tyrosine kinases and thus suppresses the production of proinflammatory cytokines such as tumour necrosis factor $\alpha(\mathrm{TNF} \alpha)$. Suppression of a TNF $\alpha$ induced cellular response may be one of the mechanisms of leflunomide efficacy in RA. ${ }^{9}$ In addition, such a mechanism could explain its contribution to this reactivation of lupus rash. ${ }^{10}$ The recent cases of lupus-like syndrome induced by anti-TNF treatment favour such a hypothesis. ${ }^{11-13}$ However, such skin manifestations were not seen when the patient was receiving infliximab combined with methotrexate for four infusions over 3 months. This observation suggests that leflunomide made a direct contribution, in line with the worsening when a higher dose was used.

An effect on the Th1/Th2 balance was evaluated by testing the systemic immune response with a bioassay based on the production of IFN $\gamma$ by PBMC. In this bioassay, patients with active RA showed a decreased production of IFN $\gamma$ in response to IL12 and IL18. This is in line with the decreased response seen in RA blood, contrasting with an increase in the joint. In contrast, at the time she had a typical lupus rash, and our patient's PBMC showed an increased production of IFN $\gamma$ in response to IL12 and particularly to IL12 + IL18 (fig 2) 
compared with controls. The picture was thus exactly opposite to that seen in RA.

In RA, the systemic defect of IFN $\gamma$ production in RA blood compared with controls appears to result from a reduced response to the Thl inducing cytokines IL12 and IL18. This situation could contribute to the increased incidence of tuberculosis, particularly after TNF inhibition. In this patient, the opposite picture was observed, with an increased response to cytokines inducing Thl. This observation is in line with an effect of leflunomide on the Th1/Th2 balance. In this case, the genetic (B8, DR3) and disease background (primary Sjögren's syndrome and not RA) may explain the appearance of lupus skin manifestations.

\section{Authors' affiliations}

D Gensburger, M Kawashima, H Marotte, P Miossec, Departments of Immunology and Rheumatology and Hospices Civils de Lyon-bioMérieux Research Unit on Rheumatoid Arthritis, Hôpital Edouard Hérriot, Lyon, France

J Kanitakis, Department of Dermatology, Hôpital Edouard Hérriot, Lyon, France

Correspondence to: Professor P Miossec, Clinical Immunology Unit, Departments of Immunology and Rheumatology, Hôpital Edouard Hérriot, 69437 Lyon Cedex 03, France; miossec@univ-lyon1.fr

Accepted 26 March 2004

\section{REFERENCES}

1 Strand V, Cohen S, Schiff M, Weaver A, Fleischmann R, Cannon G, et al. Treatment of active rheumatoid arthritis with leflunomide compared with placebo and methotrexate. Leflunomide Rheumatoid Arthritis Investigators Group. Arch Intern Med 1999;159:2542-50.

2 Mladenovic V, Domljan Z, Rozman B, Jajic I, Mihajlovic D, Dordevic J, et al. Safety and effectiveness of leflunomide in the treatment of patients with active rheumatoid arthritis. Results of a randomized, placebo-controlled, phase II study. Arthritis Rheum 1995;38:1595-603.

3 Rozman B, Praprotnik S, Logar D, Tomsic M, Hojnik M, Kos-Golja M, et al. Leflunomide and hypertension. Ann Rheum Dis 2002;61:567-9.

4 Chan AT, Bradlow A, McNally J. Leflunomide induced vasculitis-a doseresponse relationship. Rheumatology (Oxford) 2003;42:492-3.

5 McCoy CM. Leflunomide-associated skin ulceration. Ann Pharmacother 2002;36:1009-11.

6 Carulli MT, Davies UM. Peripheral neuropathy: an unwanted effect of leflunomide? Rheumatology (Oxford) 2002;41:952-3.

7 Kawashima M, MP. Decreased response to IL12 and IL18 of blood cells in rheumatoid arthritis. Arthritis Res Ther 2004;6:R39-45.

8 Breedveld FC, Dayer JM. Leflunomide: mode of action in the treatment of rheumatoid arthritis. Ann Rheum Dis 2000;59:841-9.

9 Manna SK, Mukhopadhyay A, Aggarwal BB. Leflunomide suppresses TNF-induced cellular responses: effects on NF-kappa B, activator protein-1, c-Jun N-terminal protein kinase, and apoptosis. J Immunol c-Jun N-terminal pro

10 Rubin RL. Etiology and mechanisms of drug-induced lupus. Curr Opin Rheumatol 1999;11:357-63.

11 Ali Y, Shah S. Infliximab-induced systemic lupus erythematosus. Ann Intern Med 2002; 137:625-6.

12 Carlson E, Rothfield N. Etanercept-induced lupus-like syndrome in a patient with rheumatoid arthritis. Arthritis Rheum 2003;48:1165-6.

13 Shakoor N, Michalska M, Harris CA, Block JA. Drug-induced systemic lupus erythematosus associated with etanercept therapy. Lancet 2002;359:579-80. 